STOLLER, J.: Geologisch-agronomische Karte der Gegend östlich von Verden a. d. Aller nebst Erläut. - Pr. geol. Landesanst. Berlin 1910.

TRELA, J.: Interglacial in Samostrzelniki bei Grodno in Polen. - Polska Akad. Umiej., Starunia Nr. 9, Krakau 1935. - Pollen Analysis of the interglacial formations in Olszewice. - Spraw. Kom. Fizjogr. Pol. Akad. Um. 64, Krakau 1929.

WEBER, C. A.: Utber die fossile Flora von Honerdingen und das nordwestdeutsche Diluvium. - Abh. Nat. Ver. Bremen 13, 1896.

WOLDSTEDT, P.: Über ein Interglazial bei Zweidorf (Braunschweig). Mit einem Beitrag von K. VON BÜLOW. - Z. deutsch. geol. Ges. 82, 1930. - Über die Ausdehnung der letzten Vereisung in Norddeutschland und über die Stellung des Warthe-Stadiums in der norddeutschen Eiszeitgliederung. - Ber. Reichsamt f. Bodenforsch. Wien 1942. - Über die stratigraphische Stellung einiger wichtiger Interglazialbildungen. - Z. deutsch. geol Ges. 99, 1949.

\title{
Das Interglazial von Wallensen im Hils
}

\section{Glaziale Umlagerungen interglazialer Moore}

\author{
Von Paul W. Thom son-Liblar. Mit 3 Abb.
}

Die Hilsmulde dürfte vielleicht das schönste geologische Modell im nordwestdeutschen Raume sein. Ihre geologische Bedeutung ist aber damit nicht erschōpft. Salztektonisch bedingte Senkungserscheinungen haben hier Schichtenfolgen aus verschiedenen Abschnitten des Tertiärs und Quartärs, z. T. übereinander, in wundervoller Form erhalten.

Hier befindet sich das bekannte Braunkohlenlager. von Wallensen, über dessen Alter bis jetzt keine Klarheit herrschte. Der umgelagerte sekundäre Pollen dieser Braunkohle spielt in der Hangendschichte des Interglazials eine Rolle. Ich habe hier 2 Profile, gegen 70 Einzelproben, untersucht. Außer allen im Quartär auftretenden Pollenformen, auch dem von Fagus, der im Interglazial fehlt, Ilex, cf. Hedera u. a. tritt hier der Pollen typischer tertiärer Arten und Gattungen auf. Der Pinus cf. haploxylon-Pollen ist häufiger als der von Pinus silvestris. Nicht selten sind große pinusartige Pollenkörner, die vielleicht der Gattung Keteleeria angehören dürften, neben den von Abies und Picea vorhanden. Kleine nur $70 \mu$ große Piceapollenkörner dürften von der Picea omorica oder einer nahe verwandten Art stammen. Sehr häufig, oft vorherrschend ist der Pollen der Gattung Tsuga, sowohl der vom Tsuga canadensis-, wie der vom Tsuga heterophylla-Typus. Im ganzen lassen sich 4 Tsugaformen unterscheiden. Ich habe hier Frequenzen bis $48 \%$ beobachtet. Die Tsugaarten dürften neben den Fichten und Tannen zu den häufigsten Waldbäumen dieser Zeit gehört haben. Der sehr charakteristische Pollen von Sciadopitys ist sehr verbreitet, wie auch der vom Sequoiatypus, der wohl durch lokale Bestände bedingt (Holzfunde, bestimmt von R. KRÄUSEL) in einzelnen Proben in Massen auftreten kann. Als Pollenlieferant könnte hier auch die Gattung Cryptomeria in Betracht gezogen werden. Dasselbe gilt auch für ungflügelte, glatte Coniferenpollenkörner, die von Taxodiaceen, wie Taxodium u. a., und von Cupressineen, wie Thuja, Chamaecyparis $u$. a. herrühren dürften. Von tertiären Laubbäumen tritt hier der Nyssapollen in mindestens 2 Formen in einzelnen Proben mit Frequenzen von 25\% und mehr auf, die wohl durch Lokalbestände bedingt sind. Regelmäßig ist auch der Pollen von Liquidambar anzutreffen. Dasselbe gilt auch für Carya und besonders für Pterocarya, während der von Juglans sehr selten ist. Nur ganz zerstreut sind Pollenformen von Castanea u. a. anzutreffen. Die typischen miozänen Leitformen fehlen praktisch. 
Wir haben es hier mit derselben Pollenzusammensetzung zu tun,wie sie in den Kohlenflözen im Hangenden des Limonitsandsteins im Verbande der unteren Kaolinsande anzutreffen ist (THOMSON 1949). Das Braunkohlenlager von Wallensen im Liegenden des Interglazials dürfte somit dem Pliozän angehören und der Reuver-Stufe entsprechen oder vielleicht etwas älter sein.

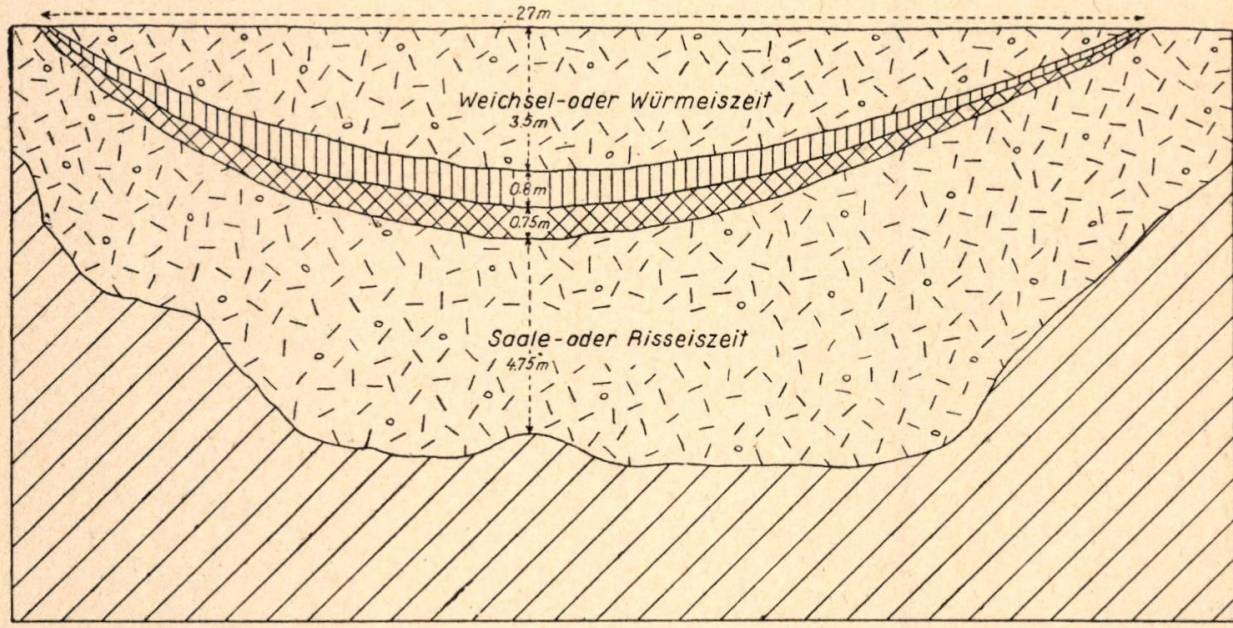

XXX Interglaziale Gyltjo

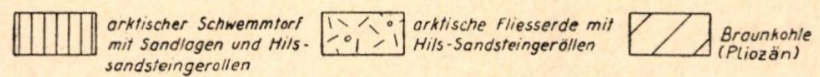

Abb. 1: Schematische Skizze der Lagerungsverhältnisse des Intenglazials von Wallensen.

Im Hangenden dieser pliozänen Braunkohle sind nun seit langem die Ablagerungen eines Interglazials bekannt (MENZEL 1903). Leider hat sich durch den fortgesetzten Abbau das Profil soweit verändert, daß eine unmittelbare Verbindung des heutigen Profils mit dem von MENZEL beschriebienen nicht mehr möglich ist. Im Zusammenhang mit den Untersuchungen des Braunkohlenlagers habe ich diesen Aufschluß im Jahre 1947 besucht, das erste Mal zusammen mit P. WOLDSTEDT. Dabei habe ich eine Serie von zusammenhängenden Schlitzproben alle $5 \mathrm{~cm}$ im unteren autochthonen Teil und alle $10 \mathrm{~cm}$ im oberen allochthonen Teil des Interglazials entnommen, die dem Diagramm Nr. III (Abb. 3) zugrunde liegen. H. GROSS hat im Herbst 1946 eine Probenserie eingesammelt, die ich ebenfalls bearbeitet habe (Diagramm Nr. I, Abb. 2). Zähltabelle Nr. II sind die untersten von H. VON GAERTNER mitgebrachten Proben. Alle 3 Profile stammen von der Nordostwand des Aufschlusses. Durch den fortgesetzten Abbau ist es aber bedingt, daß sie nicht genau von ein und derselben Stelle entnommen sind. Wie wir später sehen werden, dürfte das Profil Nr. III dem Ufer näher gelegen haben, als das Profil Nr. I. Hier sind Schichtenlücken (durch Erosion bedingt) und Umlagerungen von Uferbildungen (Schwemmtorf), die im Profil Nr. I fehlen, zu erkennen. Solche Erscheinungen sind an den Ostufern von Seen für Gebiete mit vorherrschenden Westwinden sehr bezeichnend.

Aus beiden Diagrammen geht eindeutig hervor, daß wir es mit dem letzten Interglazial - Riß-Würm (Saale-Weichsel) - zu tun haben. Sowohl im Hangenden wie im Liegenden des Interglazials sind „moränenartige“ Bildungen vorhanden (vgl. Abb. 1). Es sind das in Lehm eingebettete Brocken des Hilssand- 

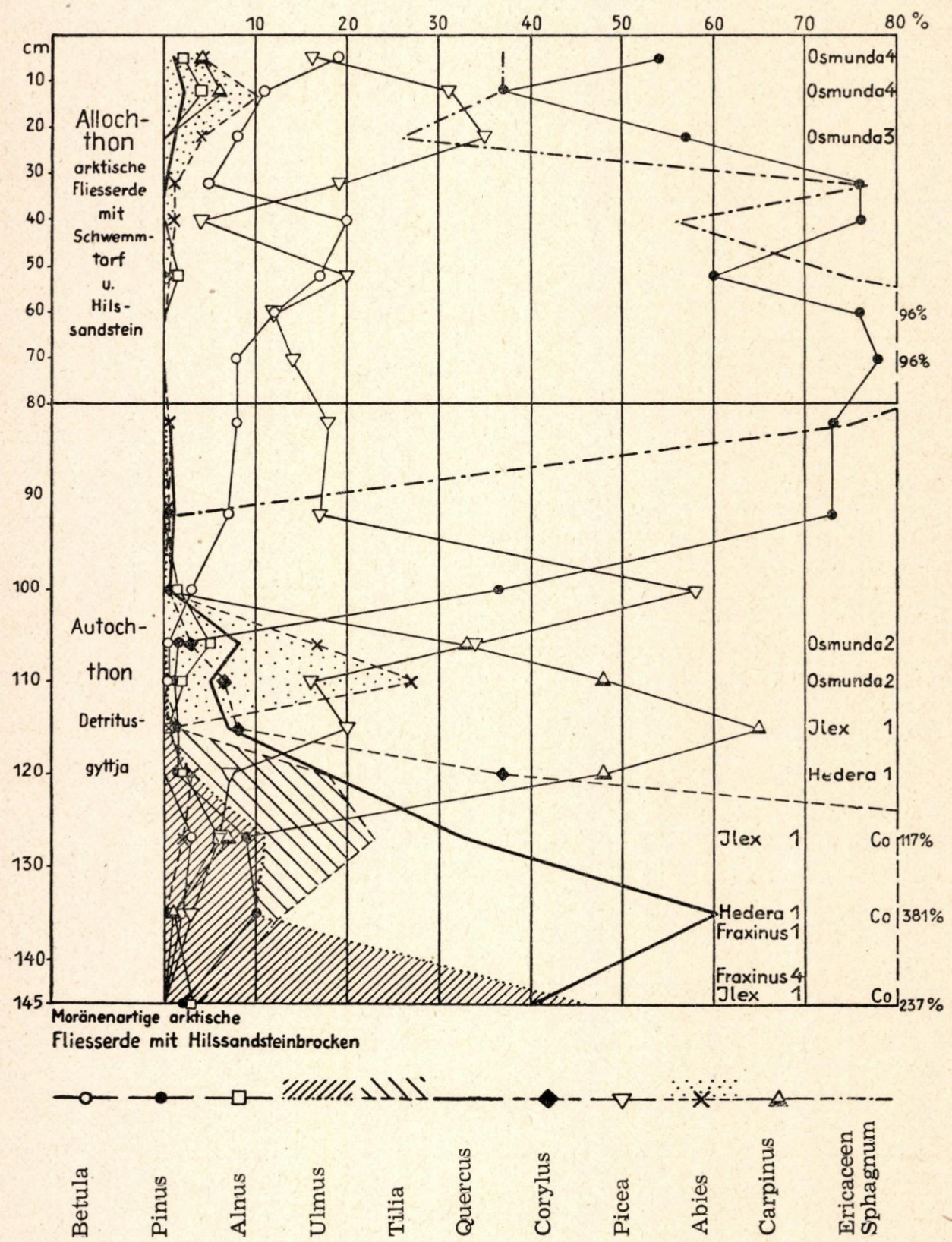

Abb. 2: Pollendiagramm I des Interglazials von Wallensen.

\begin{tabular}{|c|c|c|c|c|c|c|c|c|c|c|c|c|}
\hline & 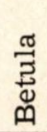 & 壳 & 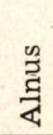 & 号 & 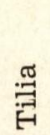 & 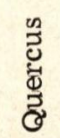 & 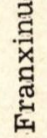 & 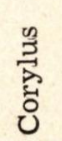 & 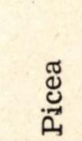 & $\frac{\mathscr{D}}{\frac{0}{4}}$ & U్ & 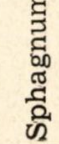 \\
\hline Basis & 5 & 20 & 3 & 27 & 11 & 32 & 2 & 232 & Spur & - & - & - \\
\hline$+30 \mathrm{~cm}$ & 2 & 8 & 0,5 & 28 & 2 & 58 & 2 & 506 & Spur & - & - & - \\
\hline
\end{tabular}

Zähltabelle Nr. II des Interglazials von Wallensen. 
steins, die nur als Schuttstrom von $\mathrm{S}$ her gekommen sein können. Nordisches Material und Feuersteine fehlen hier. Der hangende Teil dürfte demnach eine Fließerdebildung der Würm- oder Weichseleiszeit darstellen, der liegende der Riß- oder Saaleeiszeit (im erweiterten Sinne, das Warthestadium mit einbegriffen) angehören.
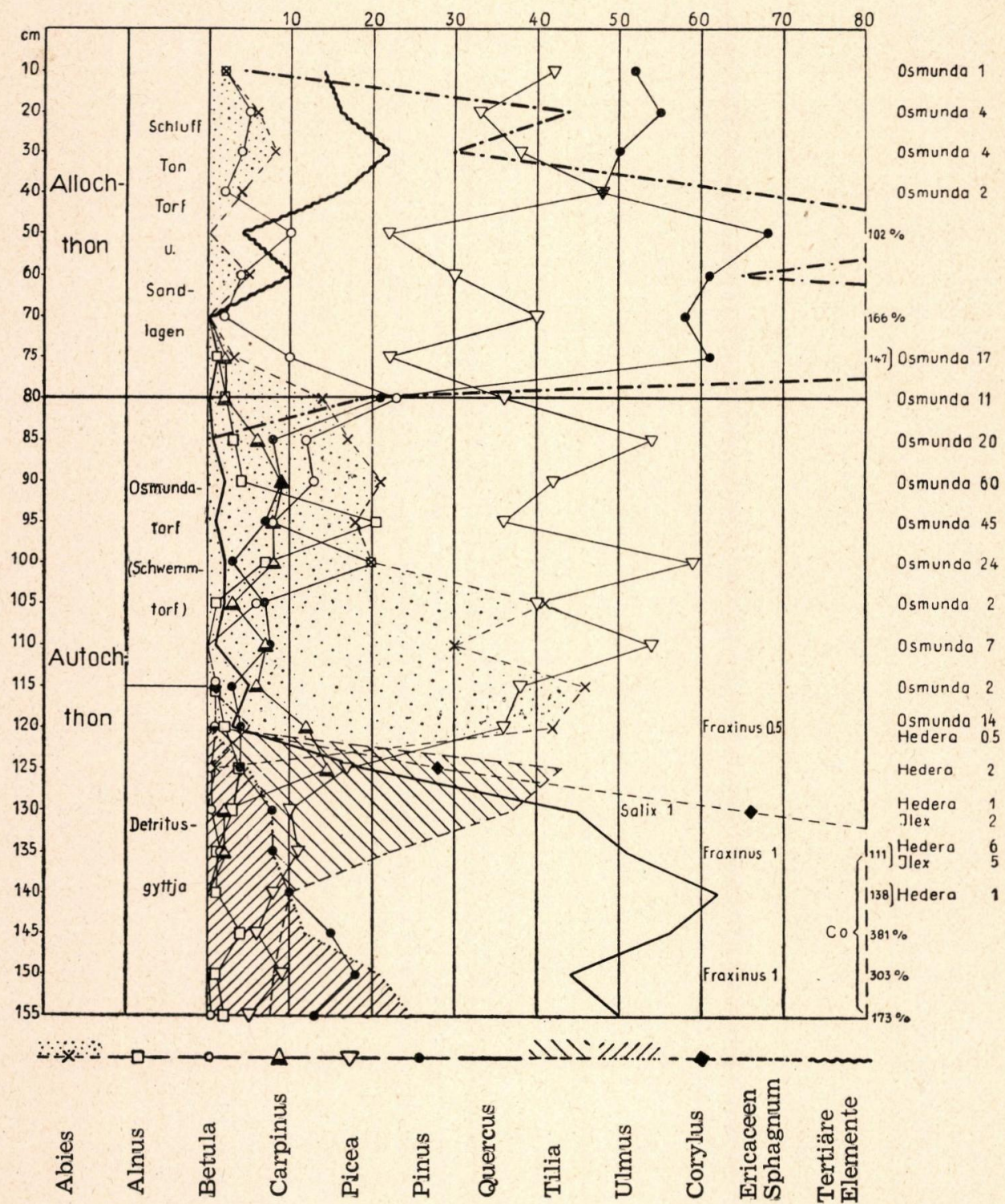

Abb. 3: Pollendiagramm III des Interglazials von Wallensen.

Im Diagramm Nr. III ist in der Kurve des tertiären Pollens nur der eindeutig bestimmte aufgenommen worden. Ein Teil des Pinus-, Picea- und Abiespollens, wie auch des durch Korrosion unkenntlichen Pollens dürfte ebenfalls aus dem Tertiär stammen.

Die wirkliche Menge des tertiären Pollens ist wesentlich größer, sie dürfte mindestens das Doppelte betragen. 
Weiter im N des Grubenaufschlusses tritt echte Grundmoräne mit nordischem Material und Feuersteinen im Hangenden der Braunkohle zutage. Die Frage, ob diese Grundmoräne noch der Riß- oder Saaleeiszeit angehört, oder schon der Mindel- oder Elstereiszeit, kann nur durch Geschiebeuntersuchungen, die noch nicht gemacht sind, entschieden werden.

Im Jahre 1947 konnte man vom Südrande der Grube aus an der Nord- und Ostwand im Abraume der Braunkohle die Ablagerungen von 2-3 Eiszeiten, ein interglaziales und ein spät- und postglaziales Moor- und Seeprofil mit einer schönen Allerödgyttja überblicken. Das allerödzeitliche Alter des unteren Gyttjalagers im spät- und postglazialen Profil südlich des interglazialen habe ich pollenanalytisch feststellen können. F. FIRBAS hat hier bei einem späteren Besuch die für die westdeutschen Allerödbildungen bezeichnenden Tuffschichten gefunden.

Die Interglazialablagerungen im Abraume der Kohle sind im unteren, mehr oder weniger autochthonen Teil im Diagramm Nr. I (Abb.2) und im Diagramm Nr. III (Abb.3) bis zum Abiesgipfel in beiden Profilen eine Detritusgyttja, die in den untersten Schichten stärker tonhaltig ist. Bis zum Carpinusgipfel stimmen beide Diagramme völlig überein. Sie beginnen, wie auch das Profil Nr. II, von dem nur die untersten Schichten vorliegen, mit einer Eichenmischwaldphase, in der die Eiche als Waldbaum vorherrscht und die Ulme reichlich vertreten ist. Der Haselpollen erreicht hier Frequenzen von über $300 \%$. Es handelt sich um den älteren oder genau genommen den mittleren Abschnitt der Wärmezeit des letzten Interglazials. F. FIRBAS hat in diesen Schichten auch die sehr bezeichnenden Samen der Gattung Brasenia festgestellt (F. FIRBAS, schriftl. Mitt.). Die unteren vor- und frühwärmezeitlichen Schichten scheinen überall zu fehlen. Die Absenkungserscheinungen, die zur Bildung des Seebeckens geführt haben, haben erst während der Wärmezeit die nötigen Beträge erreicht. Als Ursache könnte eine durch Verschwinden des rißeiszeitlichen Bodenfrostes bedingte Salzauslaugung oder Abwanderung in Betracht gezogen werden.

Der charakteristische Lindengipfel am Ende der Wärmezeit ist auch in beiden Diagrammen gut ausgebildet. Sehr bezeichnend ist für diesen Abschnitt das Auftreten großer Tiliapollenkörner, die wohl Tilia platyphyllos angehören dürften. In beiden Profilen tritt hier der sehr charakteristische Pollen von Ilex auf. Gleichzeitig tritt eine Pollenform auf, die mit großer Wahrscheinlichkeit der Gattung Hedera angehört. Das gleichzeitige Auftreten von Ilex, Tilia cf. platyphyllos und Hedera dürften einen guten Klimaindikator für diesen Abschnitt der Wärmezeit darstellen. (Nach F. FIRBAS - mündl. Mitt. - ist der Pollen der Gattung Lysimachia dem von Hedera ähnlich.) Die übrigen Nichtbaumpollen sind im wärmezeitlichen Abschnitt dieses Diagramms von nebensächlicher Bedeutung. Neben dem Auftreten von Nymphaea, Nuphar, cf. Potamogeton und Farnsporen (meist ohne Exospor, wohl Aspidium thelypteris) ist hier in $150 \mathrm{~cm}$ ein Pollenkorn cf. Succisa notiert worden.

Im Profil Nr. I folgt nun der typische Carpinusgipfel, der in dieser Interglazialzeit sogar noch in Estland nachzuweisen ist (THOMSON 1941). In dem sicher ufernäheren Profil Nr. III sind diese Schichten z. T. abgetragen worden. Es könnte sich hier um eine durch Schwankungen des Seespiegels bedingte Abtragung handeln. Wir hätten es also dann im Abschnitt des Carpinusgipfels im Profil Nr. III mit einer Erosiondiskordanz im Kleinen zu tun.

Der Abiesgipfel ist wieder im Profil Nr. III stärker entwickelt. Hier dürfte eine Uferbildung oder umgelagerter Schwemmtorf vorliegen, der massenhaft Sporen von Osmunda und Farntracheiden enthält (Osmundatorf). Es wäre denk- 
bar, daß diese Bildung durch Umlagerung von Ufertorfen bei einem Seespiegelanstieg entstanden sein könnte. Dadurch ist der Abiesgipfel im Profil Nr. III unverhältnismäßig in die Länge gezogen.

Außer der Osmundaspore treten hier noch Polypodiaceensporen und Sphagnumsporen in geringen Mengen auf. Hier habe ich auch Pollenkörner von cf. Viburnum und cf.Ribes notiert. Große (ca. $80 \mu$ ), stark gefaltete Pollenkörner könnten vielleicht der Gattung Larix angehören, wenn auch eine exakte Bestimmung nicht möglich ist. Dieser Osmunda-Schwemmtorf im Profil Nr. III ist trotz der hier vorhandenen Umlagerungserscheinungen doch eine mehr oder weniger autochthone Bildung.

Von $80 \mathrm{~cm}$ an beginnt in beiden Profilen nach oben hin eine ausgesprochene allochthone Serie. Schluffe und Tone mit Schwemmtorf wechseln hier mit Sandlagen ab, größere oder kleinere Brocken des Hilssandsteins treten auf. In den untersten $10 \mathrm{~cm}$ ist noch Material von Osmundatorf mit Abiespollen vorhanden, dann folgt in beiden Profilen eine Schichtenfolge, die in großen Mengen Hochmoorelemente enthält. Besonders im Profil Nr. I sind hier viel Sphagnumblätter und Eriophorum vaginatum-Scheidenepidermen vorhanden. In beiden Profilen tritt der Ericaceenpollen zusammen mit Sphagnumsporen in großen Mengen auf (siehe die in beiden Diagrammen übereinstimmende Kurve der Ericaceenpollentetraden und Sphagnumsporen als Summe). Unter den Ericaceen spielt Calluna eine große Rolle. Von den Waldbäumen erreicht die Kiefer ihr Maximum (wohl aus Waldhochmooren und Hochmoorrandzonen umgelagert). Wir haben es hier mit einer Sedimentation zu tun, die zusammen mit dem Abbau der in der Wärmeund besonders Nachwärmezeit entstandenen Moore unter arktischen Klimabedingungen mit gleichzeitiger Bildung von Palsen oder Torfhügeln entstanden ist.

Weiter nach oben zu sinkt in beiden Profilen die Pinuskurve und die der Sphagnumsporen und Ericaceenpollentetraden. Picea und Abies beginnen eine größere Rolle zu spielen. Osmundasporen und gelegentlich Carpinus und Quercus treten auf. Die Abtragung fördert jetzt Material auf tieferen Torfschichten der durch arktisches Klima zerstörten Moore zutage. Gleichzeitig beginnt auch der umgelagerte tertiäre (pliozäne) Pollen in größeren Mengen zu erscheinen. Im Diagramm Nr. III ist eine Kurve des eindeutig aus dem liegenden Pliozän aufgearbeiteten Pollens eingezeichnet worden, wie Liquidambar, Nyssa, Carya, Pterocarya, cf. Rhus, Pinus haploxylon, Sequoia, cf. Taxodium (Pol. hiatus), Tsuga cf. heterophylla und Tsuga canadensis, Sciadopitys u. a. Ein großer Teil der Pinus-, Picea- und Abiespollenkörner, wie auch die schlecht erhaltenen und nicht näher bestimmten dürften hier ebenfalls aus dem Tertiär stammen. So dürfte die Gesamtsumme des tertiären Pollens viel größer sein, als die in der Kurve dargestellte, die nur die Summe eindeutig tertiärer Elemente enthält. Gramineen, Cyperaceen, Umbelliferen, Compositen u. a. Pollenleörner, Farnsporen und Lycopodiumsporen kommen in der allochthonen Serie gelegentlich vor. Sie spielen aber eine unwesentliche Rolle.

Diese Pollenführung kann, wenn man den tertiären Pollen nicht kennt und das Sediment nicht berücksichtigt, leicht ein zweites Wärmemaximum vortäuschen und dürfte oft als solches gewertet worden sein (JESSEN und MILTHERS, 1928).

Im Interglazial von Ringen bei Dorpat, Estland (Rôngu bei Tartu) hat $\mathrm{K}$. ORVIKU 1939 ein eigenartiges Sediment beschrieben, das aus Gyttjageröllen, die die Größe einer Nuß erreichen können, und Schwemmtorf besteht. Ich habe darin sowohl Hochmoor- wie Niedermoorelemente festgestellt und nachweisen können, daß die Gyttjagerölle aus einer ält. Schicht stammen (THOMSON, 1941). 
Dieser umgelagerte allochthone Torf erreicht hier eine Mächtigkeit, die das autochthone Interglazial um ein Mehrfaches übertrifft. Ich bin mir damals über die Entstehung dieser Bildungen nicht im klaren gewesen. Es handelt sich hier ebenfalls um eine arktische Zerstörung wärme- und nachwärmezeitlicher Moore, wohl verbunden mit Palsenbildungen, wie ich sie nun im Hils nachweisen kann. Wenn diese Zerstörung, die mit der Bildung von Torfhügeln oder Palsen verbunden ist; tiefere Schichten in einem späteren Stadium ergreift, so wird im Sediment durch Auftreten von umgelagerten wärmezeitlichen und nachwärmezeitlichen Pollen, auch von Samen wie Brasenia u. a., ein zweites Wärmemaximum vorgetäuscht. P. WOLDSTEDT (1949) hat darauf schon hingewiesen. Für das Interglazial von Wallensen im Hils dürfte der Beweis für diese Erscheinung erbracht worden sein. Heute erleben wir in der Arktis nur den Abbau der in der postglazialen Wärmezeit entstandenen geringmächtigen Moore mit der Bildung von 5 und mehr Meter hohen Torfhügeln oder Palsen, wie im zentralen Lappland. Am Ende eines Interglazials muß dieses Phänomen von ganz wesentlich größerem Ausmaße gewesen sein, da hier die arktische Umlagerung mächtige Torflager erfaßte. Diese Umlagerung dürfte z. T. in Form von Mullwehen stattgefunden haben.

Literaturverzeichnis

1. IVERSEN, I.: Sekundäre Pollen als Fehlerquelle. Verh. III. Int. Quartär. Konf. Wien (1936) S. 225. 1938.

2. JESSEN, K. und MILTHERS, V.: Stratigraphical and paleontological studies of interglacial fresh water deposits in Jutland and Northwest Germany. Danm. Geol. Unders. II. 48. 1928.

3. MENZEL, H.: Beiträge zur Kenntnis der Quartärbildungen im südl. Hannover. Die Interglazialschichten von Wallensen in der Hilsmulde. - Jahrb. d. Pr. Geol. Landesanstalt. 24. 1903.

4. ORVIKU, K.: Mitteilungen über das Interglazial von Ringen-Rôngu (estnisch). Esti Loodus. Nr. 1. Dorpat-Tartu. 1939.

5. THOMSON, P. W.: Die Klima- und Waldentwicklung des von K. ORVIKU entdeckten Interglazials von Ringen beim Dorpat. - Z. deutsch. geol. Ges. 93. 1941.

6. - Beitrag zur Mikropaläontologie und Waldgeschichte des Neogens von Niedersachsen und Schleswig-Holstein. - N. Jahrb. f. Min. etc. Monatshefte. Abt. B. 1949. 1948.

7. WOLDSTEDT, P.: Über die stratigraphische Stellung einiger wichtiger Interglazialvorkommen im Randgebiet dẹr nordeuropäischen Vergletscherung. - Z . deutsch. geol. Ges. 99. 1949.

Manuskr. eingegangen Ende 1947.

\title{
Uber den heutigen Stand der Pollenuntersuchungen als Hilfsmittel der Quartärforschung
}

\author{
Von F. Firbas, Göttingen
}

Die Bedeutung der Pollenuntersuchungen für die Quartärforschung berubt im wesentlichen auf drei Umständen: Sie sind zunächst der zur Zeit wichtigste Weg für die Rekonstruktion der vergangenen Vegetation, die für den Botaniker der Gegenstand seiner Bemühungen ist, für andere Wissenschaften aber ein wichtiges Stück der Landschaft, z. B. ein Stück der Umwelt des urgeschichtlichen Menschen. Die weitgehende Abhängigkeit der Pflanzendecke vom Klima bringt es weiter mit sich, daß jede vegetationsgeschichtliche Untersuchung eine klima- 